\title{
Lens Exposure during Brain Scans Using Multidetector Row CT Scanners: Methods for Estimation of Lens Dose
}

\footnotetext{
S. Suzuki

BACKGROUND AND PURPOSE: Some recent studies on radiation lens injuries have indicated much

S. Furui

T. Ishitake

T. Abe lower dose thresholds than specified by the current radiation protection guidelines. The purpose of this research was to measure the lens dose during brain CT scans with multidetector row CT and to assess methods for estimating the lens dose.

H. Machida

R. Takei

K. Ibukuro

MATERIALS AND METHODS: With 8 types of multidetector row CT scanners, both axial and helical scans were obtained for the head part of a human-shaped phantom by using normal clinical settings with the orbitomeatal line as the baseline. We measured the doses on both eyelids by using an RPLGD during whole-brain scans including the orbit with the starting point at the level of the inferior orbital rim. To assess the effect of the starting points on the lens doses, we measured the lens doses by using 2 other starting points for scanning (the orbitomeatal line and the superior orbital rim).

T. Kidouchi

RESULTS: The CTDIvols and the lens doses during whole-brain CT including the orbit were 50.9-113.3 $\mathrm{mGy}$ and $42.6-103.5 \mathrm{mGy}$, respectively. The ratios of lens dose to CTDIvol were $80.6 \%-103.4 \%$. The lens doses decreased as the starting points were set more superiorly. The lens doses during scans from the superior orbital rim were $11.8 \%-20.9 \%$ of the doses during the scans from the inferior orbital rim.

CONCLUSIONS: CTDIvol can be used to estimate the lens dose during whole-brain CT when the orbit is included in the scanning range.

\begin{abstract}
ABBREVIATIONS: $\mathrm{CTDI}=\mathrm{CT}$ dose index; CTDIvols $=$ volume $\mathrm{CT}$ dose indices; $\mathrm{D}_{\mathrm{IOR}}=$ lens dose during whole-brain scanning from the inferior orbital rim; $D_{\mathrm{OM}}=$ lens dose during scanning from the orbitomeatal line; $D_{\text {SOR }}=$ lens dose during scanning from the superior orbital rim; ICRP = International Commission on Radiological Protection; $\mathrm{mAs}_{\text {eff }}=\mathrm{mAs}$ divided by helical pitch;
\end{abstract} RPLGD = radiophotoluminescent glass dosimeter
}

T he eye lens is one of the most radiosensitive tissues. According to 1990 recommendations of the ICRP, ${ }^{1}$ the thresholds in a single brief exposure for detectable opacities and visual impairment (cataract) are 0.5-2.0 and 5.0 Sv, respectively. In highly fractionated or protracted exposures, the threshold is $5 \mathrm{~Sv}$ for detectable opacities and $>8$ Sv for cataracts. However, a number of recent studies have supported lower thresholds for radiation-induced lens injuries. ${ }^{2-6}$ Some authors have suggested that the risk of cataract increases with increased radiation dose without a threshold. ${ }^{2,3}$ Given these data, the ICRP referred to the need for a detailed revaluation of the radiosensitivity of the lens. ${ }^{7}$

During brain CT scans, the lens is irradiated indirectly and/or directly. With single-detector row CT, the evaluation of the posterior cranial fossa had been limited by streak artifacts caused by the thick irregular bone of the skull base. ${ }^{8}$ On the other hand, multidetector row CT provides better images with fewer artifacts in the posterior cranial fossa. ${ }^{8}$ However,

Received June 15, 2009; accepted after revision October 1.

From the Department of Radiology (S.S., S.F., A.W., T.K., Y.N.), Teikyo University School of Medicine, Tokyo, Japan; Departments of Environmental Medicine (T.I.) and Radiology (T.A.), Kurume University School of Medicine, Fukuoka, Japan; Department of Radiology (H.M.), Tokyo Women's Medical University, Medical Center East, Tokyo, Japan; Radiology Department (R.T.), The Cardiovascular Institute Hospital, Tokyo, Japan; and Department of Radiology (K.I.), Mitsui Memorial Hospital, Tokyo, Japan.

Please address correspondence to Shigeru Suzuki, MD, Department of Radiology, Teikyo University School of Medicine, 2-11-1 Kaga, Itabashi-ku, Tokyo, 173-8605, Japan; e-mail: s-suzuki@med.teikyo-u.ac.jp

DOI 10.3174/ajnr.A1946 the orbit is included in the scanning range during whole-brain $\mathrm{CT}$, including the posterior cranial fossa, if the orbitomeatal line is used as a baseline. ${ }^{9}$ Considering the above studies supporting lower thresholds for radiation-induced lens injuries, we believe the exposure to the lens during brain CT scans with multidetector row CT should be re-evaluated. However, it is difficult to estimate the lens dose during brain CT in individual cases because the dose varies considerably due to differences in the type of CT scanners and scan settings. Bauhs et $\mathrm{al}^{10}$ reported that the CTDI can be used theoretically to estimate the average dose from multiple scans with table increments.

Table 1 shows the formulas for CTDI and its variations. However, the surface dose is not constant along the z-axis in the scanning range of whole-brain CT. The dose profile along the $\mathrm{z}$-axis has multiple peaks according to the number of axial scans. ${ }^{10}$ Even with helical scanning (and of course with axial scanning), the doses near the starting and end points are lower than the dose of the central portion of the scanning range along $\mathrm{z}$-axis due to the presence of less scatter radiation. The dose is not constant in the $\mathrm{x}-\mathrm{y}$ plane either due to a bow-tie filter. A bow-tie filter is automatically used for brain scans in many multidetector row CT scanners to reduce the peripheral dose, and it affects the surface doses. ${ }^{11}$ Moreover, the homogeneous polymethylmethacrylate phantom for CTDI measurement does not simulate the different tissue types and heterogeneities of a real patient. These factors preclude CTDI from being the same as tissue dosimetry in a real patient. To our knowledge, there have been no reports describing the re- 


\begin{tabular}{|c|c|c|}
\hline Dose Index & Formula & Notes \\
\hline CTDI & $C T D I=\frac{1}{T} \int_{-\infty}^{+\infty} D(z) d z$ & $\begin{array}{l}\mathrm{T} \text { is the nominal width of a section and } \mathrm{D}(\mathrm{z}) \text { is the dose } \\
\text { at point } z \text { parallel to the } z \text {-axis }\end{array}$ \\
\hline CTDI measured $>100 \mathrm{~mm}$ & $C T D I_{100}=\frac{1}{N T} \int_{-50 m m}^{+500 m m} D(z) d z$ & $\begin{array}{l}\mathrm{N} \text { is the number of acquired sections per scan, } \mathrm{T} \text { is } \\
\text { the nominal width of each acquired section, and } \mathrm{D}(\mathrm{z}) \\
\text { is the dose at point } \mathrm{z} \text { parallel to the z-axis }\end{array}$ \\
\hline Weighted CTDI & $C T D I W=\frac{1}{3} C T D I_{100, \text { center }}+\frac{2}{3} C T D I_{100, \text { periphery }}$ & $\begin{array}{l}\mathrm{CTDI}_{100, \text { center }} \text { is the CTDI } \\
\text { of the standard CTDI phantom and CTDI } \\
\text { the CTDI } \\
\text { 100, meariphery is } \\
\text { the phantom }\end{array}$ \\
\hline CTDlvol & CTDIvol $=\frac{\text { CTDIw }}{\text { pitch }}$ & Pitch is table travel distance per rotation divided by nominal beam width \\
\hline
\end{tabular}

\begin{tabular}{|c|c|c|c|c|c|c|c|}
\hline \multirow[b]{2}{*}{ CT Scanner (number of detector rows) } & \multicolumn{3}{|c|}{ Axial Scan } & \multicolumn{4}{|c|}{ Helical Scan } \\
\hline & Collimation (mm) & $\begin{array}{l}\text { Rotation Time } \\
\text { (sec) }\end{array}$ & mAs & Collimation (mm) & $\begin{array}{l}\text { Rotation Time } \\
\text { (sec) }\end{array}$ & $\mathrm{mAs}_{\text {eff }}$ & $\begin{array}{c}\text { Helical } \\
\text { Pitch }\end{array}$ \\
\hline $\begin{array}{l}\text { Aquilion } 64 \text { (64), Toshiba Medical Systems, } \\
\text { Tokyo, Japan }\end{array}$ & $8 \times 0.5$ & 1 & 350 & $64 \times 0.5$ & 1 & 300 & 0.641 \\
\hline $\begin{array}{l}\text { LightSpeed VCT (64), GE Healthcare, Milwaukee, } \\
\text { Wisconsin }\end{array}$ & $16 \times 0.625$ & 2 & 420 & $32 \times 0.625$ & 1 & 433 & 0.531 \\
\hline LightSpeed Ultra 16 (16), GE Healthcare & $8 \times 1.25$ & 2 & 400 & $16 \times 0.625$ & 0.7 & 261 & 0.938 \\
\hline LightSpeed Ultra 8 (8), GE Healthcare & $4 \times 3.75$ & 2 & 360 & $8 \times 1.25$ & 0.8 & 256 & 0.625 \\
\hline LightSpeed QX/i (4), GE Healthcare & $4 \times 2.5$ & 2 & 400 & $4 \times 2.5$ & 0.7 & 326 & 0.75 \\
\hline $\begin{array}{l}\text { Brilliance CT } 64 \text { (64), Philips Medical Systems, } \\
\text { Best, the Netherlands }\end{array}$ & $16 \times 0.625$ & 1.5 & 450 & $64 \times 0.625$ & 0.5 & 450 & 0.423 \\
\hline MX 8000 IDT (16), Philips Medical Systems, & $16 \times 1.5$ & 1.5 & 400 & $16 \times 0.75$ & 0.75 & 400 & 0.5 \\
\hline $\begin{array}{l}\text { Somatom Sensation Cardiac } 64 \text { (64), Siemens, } \\
\text { Malvern, Pennsylvania }\end{array}$ & $12 \times 1.2$ & 2 & 380 & $20 \times 0.6$ & 1 & 422 & 0.9 \\
\hline
\end{tabular}

lationship between the patient's lens dose and CTDI during brain CT scans with multidetector row CT.

The purpose of this research was to measure the lens dose during brain CT with multidetector row $\mathrm{CT}$ and to assess methods for estimating the lens dose with CTDIvols.

\section{Materials and Methods}

\section{CT Scanning}

Using 8 types of multidetector row CT scanners, we obtained wholebrain CT scans for the head part of a Rando phantom (Phantom Laboratories, Salem, New York), which represented a 163-cm-tall and 54-kg female figure. We used both axial and helical scans for each CT scanner. Table 2 shows the scanning parameters. For each scanner, tube voltage was set at $120 \mathrm{kV}$, and the scanning parameters in Table 1 represent the normal clinical settings. We used the orbitomeatal line as the baseline. In each scanner, the CTDIvol was displayed on the console.

The starting point of the whole-brain CT scan was $15 \mathrm{~mm}$ below the orbitomeatal line, and the end point was the top of the head. The former corresponded to the level of the inferior orbital rim.

\section{Lens-Dose Measurement on Human-Shaped Phantoms}

We measured the doses on the centers of both eyelids by using a RPLGD. We regarded the average dose of both sides as the lens dose. To estimate the uncertainty in these dose assessments, we repeated the measurements 3 times. The RPLGD chip (GD-352M, Asahi Techno Glass, Shizuoka, Japan) consists of a glass element with a $1.5-\mathrm{mm}$ diameter and 12-mm length and a holder with an energy-compensation filter of $0.75-\mathrm{mm}$ tin. We annealed the glass elements of the
RPLGD for 1 hour at $400^{\circ} \mathrm{C}$ and cooled them down slowly to room temperature before the exposure. A preheating process was performed for 30 minutes at $70^{\circ} \mathrm{C}$ after the exposure, and a fully automatic system (FGD-1000, Asahi Techno Glass) was used for the readout. For calibration, we used a standard glass irradiated with $137 \mathrm{Cs}$ of gamma ray energy $(0.662 \mathrm{MeV})$ of $6 \mathrm{mGy}$. According to the data provided by the manufacturer, the coefficient of variation is $\leq 2 \%$ at $\geq 1 \mathrm{mGy}$.

\section{Effect of the Starting Point of the Scanning on Lens Dose}

To assess the effect of the starting points on the lens doses, we measured the lens doses during brain CT scanning for axial scans by using 2 other starting points of scanning (the orbitomeatal line and the superior orbital rim). The latter corresponded to the level $15 \mathrm{~mm}$ above the orbitomeatal line. The end points were the top of the head in all scans.

\section{Results}

Table 3 shows CTDIvols and the lens doses during wholebrain CT including the orbit for both axial and helical scans with each CT scanner. The CTDIvols and the lens doses were 50.9-113.3 $\mathrm{mGy}$ and 42.6-103.5 $\mathrm{mGy}$, respectively. The former tended to be larger than the latter, and the ratios of lens dose to CTDIvol were $80.6 \%-103.4 \%$.

Table 4 shows the effects of the starting point of the scanning on the lens dose. The lens doses decreased as the starting points were set more superiorly. The lens doses during scanning from the superior orbital rim were $11.8 \%-20.9 \%$ of the doses during scanning from the inferior orbital rim. The ratios 


\begin{tabular}{|c|c|c|c|c|c|c|}
\hline \multirow[b]{2}{*}{$\begin{array}{l}\text { CT Scanner (Number of } \\
\text { Detector Rows) }\end{array}$} & \multicolumn{3}{|c|}{ Axial Scan } & \multicolumn{3}{|c|}{ Helical Scan } \\
\hline & $\begin{array}{l}\text { Lens Dose } \\
\text { (mGy) }\end{array}$ & $\begin{array}{l}\text { CTDlvol } \\
\text { (mGy) }\end{array}$ & $\begin{array}{l}\text { Lens Dose/ } \\
\text { CTDlvol }\end{array}$ & $\begin{array}{l}\text { Lens Dose } \\
\text { (mGy) }\end{array}$ & $\begin{array}{l}\text { CTDlvol } \\
\text { (mGy) }\end{array}$ & $\begin{array}{l}\text { Lens Dose/ } \\
\text { CTDlvol }\end{array}$ \\
\hline Aquilion 64 (64) & $103.5 \pm 7.2$ & $113.3 \pm 0.0$ & $91.4 \pm 6.4 \%$ & $82.3 \pm 7.3$ & $96.0 \pm 0.0$ & $85.7 \pm 7.6 \%$ \\
\hline LightSpeed VCT (64) & $81.5 \pm 9.8$ & $101.1 \pm 0.0$ & $80.6 \pm 9.7 \%$ & $75.4 \pm 0.9$ & $74.3 \pm 0.0$ & $101.5 \pm 1.2 \%$ \\
\hline LightSpeed Ultra 16 (16) & $71.5 \pm 2.4$ & $85.8 \pm 0.0$ & $83.3 \pm 2.8 \%$ & $48.7 \pm 0.9$ & $59.6 \pm 0.0$ & $81.7 \pm 1.5 \%$ \\
\hline LightSpeed Ultra 8 (8) & $56.6 \pm 0.9$ & $66.5 \pm 0.0$ & $85.1 \pm 1.3 \%$ & $45.6 \pm 0.7$ & $50.9 \pm 0.0$ & $89.7 \pm 1.4 \%$ \\
\hline LightSpeed OX/i (4) & $70.2 \pm 1.5$ & $75.1 \pm 0.0$ & $93.5 \pm 2.0 \%$ & $66.7 \pm 1.4$ & $65.1 \pm 0.0$ & $102.5 \pm 2.1 \%$ \\
\hline Brilliance CT 64 (64) & $61.7 \pm 4.9$ & $72.4 \pm 0.0$ & $85.3 \pm 6.8 \%$ & $52.6 \pm 0.8$ & $50.9 \pm 0.0$ & $103.4 \pm 1.6 \%$ \\
\hline MX 8000 IDT (16) & $48.1 \pm 3.2$ & $51.8 \pm 0.0$ & $92.8 \pm 6.2 \%$ & $57.4 \pm 1.4$ & $57.0 \pm 0.0$ & $100.7 \pm 2.4 \%$ \\
\hline Somatom Sensation Cardiac (64) & $48.4 \pm 1.7$ & $58.9 \pm 0.0$ & $82.2 \pm 2.8 \%$ & $42.6 \pm 0.9$ & $51.5 \pm 0.0$ & $82.8 \pm 1.7 \%$ \\
\hline
\end{tabular}

Table 4: Effects of starting point of scanning on the lens dose

\begin{tabular}{|c|c|c|c|c|c|c|}
\hline \multirow[b]{2}{*}{$\begin{array}{l}\text { CT Scanner (Number of } \\
\text { Detector Rows) }\end{array}$} & \multicolumn{3}{|c|}{ Scans from Orbitomeatal Line } & \multicolumn{3}{|c|}{ Scans from Superior Orbital Rim } \\
\hline & $\begin{array}{c}\mathrm{D}_{\mathrm{OM}} \\
\text { (mGy) }\end{array}$ & $\begin{array}{c}D_{O M} / D_{\text {IOR }} \\
(\%)\end{array}$ & $\begin{array}{c}\mathrm{D}_{\mathrm{OM} / \mathrm{CTDlvol}} \\
(\%)\end{array}$ & $\begin{array}{l}\mathrm{D}_{\text {SOR }} \\
\text { (mGy) }\end{array}$ & $\begin{array}{c}\mathrm{D}_{\mathrm{SOR}} / \mathrm{D}_{\mathrm{IOR}} \\
(\%)\end{array}$ & $\begin{array}{c}\mathrm{D}_{\text {SOR }} / \text { CTDlvol } \\
(\%)\end{array}$ \\
\hline Aquilion 64 (64) & $89.9 \pm 4.4$ & 86.9 & 79.3 & $12.2 \pm 0.6$ & 11.8 & 10.8 \\
\hline LightSpeed VCT (64) & $64.5 \pm 1.5$ & 79.1 & 60.3 & $11.0 \pm 0.7$ & 13.5 & 10.4 \\
\hline LightSpeed Ultra 16 (16) & $57.9 \pm 0.9$ & 81.0 & 67.5 & $9.2 \pm 0.2$ & 12.9 & 10.7 \\
\hline LightSpeed Ultra 8 (8) & $52.2 \pm 1.6$ & 92.2 & 78.5 & $7.7 \pm 0.3$ & 13.6 & 11.6 \\
\hline LightSpeed $\mathrm{OX} / \mathrm{i}(4)$ & $64.6 \pm 0.6$ & 92.0 & 86.0 & $10.3 \pm 0.2$ & 14.7 & 13.7 \\
\hline Brilliance CT 64 (64) & $56.9 \pm 2.1$ & 92.2 & 78.6 & $12.9 \pm 0.6$ & 20.9 & 17.8 \\
\hline MX 8000 IDT (16) & $44.6 \pm 3.7$ & 92.7 & 86.1 & $7.1 \pm 0.3$ & 14.8 & 13.7 \\
\hline Somatom Sensation Cardiac 64 (64) & $42.2 \pm 0.4$ & 87.2 & 71.8 & $8.9 \pm 0.8$ & 8.4 & 15.2 \\
\hline
\end{tabular}

of lens dose to CTDIvol during scanning from the orbitomeatal line and from the superior orbital rim were $60.3 \%$ $86.1 \%$, and $10.4 \%-17.8 \%$, respectively.

\section{Discussion}

As pointed out in ICRP publication $103,{ }^{7}$ some recent studies on radiation lens injuries have indicated much lower dose thresholds than specified by the current radiation-protection guidelines. In a study conducted on atomic bomb survivors by Nakashima et al, ${ }^{2}$ the threshold doses for cortical cataract and posterior subcapsular cataract were $0.6 \mathrm{~Sv}$ and $0.7 \mathrm{~Sv}$, respectively. ${ }^{2}$ In another study of atomic bomb survivors, Neriishi et $\mathrm{al}^{3}$ reported that the dose threshold was 0.1 Gy for postoperative cataracts. As for protracted radiation exposures, Worgul et $\mathrm{al}^{6}$ investigated cataracts in Chernobyl clean-up workers. Their data indicated that the cumulative dose threshold for cataracts was $<700 \mathrm{mGy}$. Other recent studies of radiation cataracts in airline pilots and astronauts also supported much lower dose thresholds for cataracts than do the guidelines. ${ }^{12,13}$

Furthermore, the lens of a child is more sensitive to radiation exposure than that of an adult. ${ }^{2,14,15}$ Wilde and Sjöstrand $^{14}$ reported a clinical study of radiation-induced lens injuries among patients receiving radium irradiation to treat hemangioma in the eyelid in early childhood (age range, 1.5-13 months). In 13 of 16 untreated eyes with irradiation of 0.04-0.12 Gy, posterior subcapsular opacities $(n=12)$ or posterior subcapsular cataract $(n=1)$ was found.

In the current study with multidetector row $\mathrm{CT}$, the lens doses during 1 series of whole-brain CT scans were 50-100 mGy. Considering the data of the above-mentioned recent studies, the cumulative lens doses of several series of CT scans should not be neglected from the viewpoint of radiologic protection. In fact, a population-based study of common agerelated eye disease by Klein et al in $1993^{16}$ showed that nuclear sclerosis and posterior subcapsular opacity were significantly associated with CT scans of the head. Another study of radiation cataracts indicated a significant association between a history of $\geq 3$ diagnostic $x$-rays to the face or neck and increased risk of cataract. ${ }^{17}$

The lens dose during brain CT is affected by the type of CT scanner and the scanning settings. Therefore, it is important to estimate the lens dose during brain CT in individual cases in each institution. Bauhs et $\mathrm{al}^{10}$ reported that the CTDI can be used theoretically to estimate the average dose from multiple scans with table increments. However, the dose is not constant along the z-axis in the scanning range of whole-brain CT. During whole-brain CT by using axial scanning, multiple axial scans are needed to cover the scanning range. The dose profile along the $\mathrm{z}$-axis has multiple peaks, according to the number of axial scans. ${ }^{10}$ Even with helical scanning, the doses near the starting and end points are lower than the dose of the central portion of the scan range along the $\mathrm{z}$-axis. Scatter radiation exists outside the beam width both cranially and caudally. The central portion is exposed by both cranial and caudal scatter radiation. On the other hand, the starting or end point of the scanning receives either cranial or caudal scatter radiation. Furthermore, bow-tie filters affect the dose distribution in the $\mathrm{x}-\mathrm{y}$ plane.

According to the data of Avilés Lucas et al, ${ }^{11}$ surface dose decreases as the measurement point moves vertically away from the scanning center. In their study, the surface doses were $83 \%$ and $62 \%$ at 8 and $12 \mathrm{~cm}$ from the scanning center, respectively, compared with the dose at $2.9 \mathrm{~cm}$. Moreover, the homogeneous polymethylmetacrylate phantom for CTDI measurement does not simulate the different tissue types and heterogeneities of a real patient. ${ }^{18}$ Therefore, CTDI does not generally serve as an accurate estimate of the radiation dose to 
a point in a real patient, though it is an index of radiation dose due to CT scans.

In the current study, the ratios of lens dose to CTDIvol were $80.6 \%-103.4 \%$, and the lens doses tended to be smaller than the CTDIvols during whole-brain CT including the orbit. As mentioned above, scatter radiation decreases at the starting point of the scanning, and the peripheral dose is decreased by the bow-tie filter in the $x-y$ plane. Therefore, the lens doses during the whole-brain CT were probably smaller than the CTDIvols. When the orbits were included in the scanning range, the differences between the lens dose and CTDIvol were within $20 \%$ for both helical and axial scans with each CT scanner, with this degree of difference being acceptable for clinical use. Therefore, the lens dose can be estimated approximately by the CTDIvol, when the orbit is included in the scanning range. This method is useful to easily estimate the patient's lens dose during brain CT on the basis of CTDIvol because the values of CTDIvol are displayed on the monitors immediately after the scanning.

The lens doses decreased as the starting points were set more superiorly. The ratios of lens dose to CTDIvol were $10 \%-20 \%$ when the scanning started from the superior orbital rim. These results are in agreement with the work of Smith et al in 1998, ${ }^{19}$ who measured weighted CTDI values and lens doses during brain CT on phantoms by using single-detector row CT scanners. On the basis of their data, we calculated the ratio of lens doses to weighted CTDIs. The ratio was calculated as $88.4 \pm 12.7 \%$ during scanning including the whole orbit (baseline: orbitomeatal line or infraorbitomeatal line), while it was $13.9 \pm 4.8 \%$ during scanning excluding the orbit (baseline: supraorbitomeatal line).

Considering the lens dose during brain CT scans and the above-mentioned uncertainty about the risk of radiation lens injuries, we believe that the exposure to the lens during brain CT scans should be optimized. For dose reduction, it is fundamental to decrease CTDIvol by optimal selection of scanning parameters. However, this method has a limit, given the proposed reference level for brain CT, which was $60 \mathrm{mGy}$ by the ICRP. ${ }^{20}$ There are several additional methods to reduce the lens dose during brain CT. In the follow-up CT scans for patients without lesions in the posterior cranial fossa, exclusion of the posterior cranial fossa from the scanning range results in reduced lens dose if the orbitomeatal line is used as a baseline. The lens can be excluded from the scanning range by using a more angulated baseline than the orbitomeatal line, even when the posterior cranial fossa is scanned. ${ }^{9,21}$ Eye masks such as a bismuth-coated latex shield are also useful to reduce the lens exposure. ${ }^{22,23}$ Improvement in the CT scanner would also be desirable. With automatic tube-current modulation in the $\mathrm{x}-\mathrm{y}$ plane, decreased anteroposterior exposure with increased posteroanterior exposure should reduce the lens dose, while maintaining the image quality.

This study has some limitations. First, we used only 1 type of human-shaped phantom. The size and shape of the objects, especially the distance from the scanning center to the lens, may affect the surface dose. However, the sizes of adult heads have small individual differences. According to the data obtained on adult Japanese by Demura et al, ${ }^{24}$ the mean head lengths were $23.3 \pm 1.2 \mathrm{~cm}$ and $21.8 \pm 0.9 \mathrm{~cm}$ in men and women, respectively. Second, the CTDIvol values during brain CT vary among institutions, even with the same type of CT scanner because they are affected by the scanning parameters selected. However, CTDIvol values in the current study agreed with previous survey data. The weighted CTDI was $50.0 \pm 14.6 \mathrm{mGy}$ (dose range, $21.0-130 \mathrm{mGy}$ ) in the United Kingdom as reported by Shrimpton et al, ${ }^{25}$ while the average CTDIvols were $72.2 \mathrm{mGy}$ for adults and $42.0 \mathrm{mGy}$ for 5 - to 7-year-old children in Australia, according to Moss and McLean. ${ }^{26}$ Therefore, the results of the current study can be adapted to many conditions.

Third, CTDIvol provides the estimate of the lens dose during brain CT only when the orbit is included in the scanning range. However, the lens dose during brain $\mathrm{CT}$ including the orbit is larger than that during brain CT excluding the orbit, and dose estimation is more important for the former. Fourth, the precise risk of radiation lens injuries for brain CT is still unknown. As for the risk of radiation lens injuries at a low dose, available studies are limited at present, and many of them were conducted on atomic bomb survivors. ${ }^{2-5}$ The beam quality and dose rate differ between the exposure to patients undergoing brain CT and the exposure to the objects in these studies, and the differences will affect the risk. Future risk assessment of radiation lens injuries for diagnostic $\mathrm{x}$-rays is desirable.

In conclusion, CTDIvol can be used to estimate the lens dose during brain CT scanning, when the orbit is included in the scanning range. It is important to estimate the dose to the lens during brain CT scans and try to reduce it.

\section{References}

1. 1990 Recommendations of the International Commission on Radiological Protection. Ann ICRP 1991;21:1-201

2. Nakashima E, Neriishi K, Minamoto A. A reanalysis of atomic-bomb cataract data, 2000-2002: a threshold analysis. Health Phys 2006;90:154-60

3. Neriishi K, Nakashima E, Minamoto A, et al. Postoperative cataract cases among atomic bomb survivors: radiation dose response and threshold. Radiat Res 2007;168:404-08

4. Minamoto A, Taniguchi $\mathrm{H}$, Yoshitani N, et al. Cataract in atomic bomb survivors. Int J Radiat Biol 2004;80:339-45

5. Otake M, Neriishi K, Schull WJ. Cataract in atomic bomb survivors based on a threshold model and the occurrence of severe epilation. Radiat Res 1996;146:339-48

6. Worgul BV, Kundiyev YI, Sergiyenko NM, et al. Cataracts among Chernobyl clean-up workers: implications regarding permissible eye exposures. Radiat Res 2007;167:233-43

7. The 2007 Recommendations of the International Commission on Radiological Protection. ICRP Publication 103. Ann ICRP 2007;159-171

8. Jones TR, Kaplan RT, Lane B, et al. Single- versus multi-detector row CT of the brain: quality assessment. Radiology 2001;219:750-55

9. Yeoman LJ, Howarth L, Britten A, et al. Gantry angulation in brain CT: dosage implications, effect on posterior fossa artifacts, and current international practice. Radiology 1992;184:113-16

10. Bauhs JA, Vrieze TJ, Primak AN, et al. CT dosimetry: comparison of measurement techniques and devices. Radiographics 2008;28:245-53

11. Avilés Lucas P, Castellano IA, Dance DR, et al. Analysis of surface dose variation in CT procedures. Br J Radiol 2001;74:1128-36

12. Rafnsson V, Olafsdottir E, Hrafnkelsson J, et al. Cosmic radiation increases the risk of nuclear cataract in airline pilots: a population-based case-control study. Arch Ophthalmol 2005;123:1102-05

13. Cucinotta FA, Manuel FK, Jones J, et al. Space radiation and cataracts in astronauts. Radiat Res 2001;156:460-66

14. Wilde G, Sjöstrand J. A clinical study of radiation cataract formation in adult life following gamma irradiation of the lens in early childhood. J Ophthalmol 1997;81:261-66

15. Chen WL, Hwang JS, Hu TH, et al. Lenticular opacities in populations exposed to chronic low-dose-rate gamma radiation from radiocontaminated buildings in Taiwan. Radiat Res 2001;156:71-77

16. Klein BE, Klein R, Linton KL, et al. Diagnostic $\mathbf{x}$-ray exposure and lens opacities: the Beaver Dam Eye Study. Am J Public Health 1993;83:588-90 
17. Chodick G, Bekiroglu N, Hauptmann M, et al. Risk of cataract after exposure to low doses of ionizing radiation: a 20-year prospective cohort study among US radiologic technologists. Am J Epidemiol 2008;168:620-31

18. McNitt-Gray MF. AAPM/RSNA physics tutorial for residents: topics in CTradiation dose in CT. Radiographics 2002;22:1541-53

19. Smith A, Shah GA, Kron T. Variation of patient dose in head CT. Br J Radiol 1998;71:1296-301

20. Task Group on Control of Radiation Dose in Computed Tomography. Managing patient dose in computed tomography: a report of the International Commission on Radiological Protection. Ann ICRP 2000;30:7-45

21. Lai KF, Cheung YK, Tan CB, et al. Lens exclusion in computed tomography scans of the brain: the local practice. J HK Coll Radiol 2001;4:181-84
22. Perisinakis K, Raissaki M, Theocharopoulos N, et al. Reduction of eye lens radiation dose by orbital bismuth shielding in pediatric patients undergoing CT of the head: a Monte Carlo study. Med Phys 2005;32:1024-30

23. Hopper KD, Neuman JD, King SH, et al. Radioprotection to the eye during CT scanning. AJNR Am J Neuroradiol. 2001;22:1194-98

24. Demura S, Yamaji S, Nakada M, et al. Prediction equation for head volume of Japanese young adults. J Sports Sci 2005;23:541-48

25. Shrimpton PC, Jones DG, Hillier MC, et al. Survey of CT practice in the UK. Part 2. Dosimetric aspects. In: National Radiological Protection Board, Report NRPB-R 249. London, UK: HMSO; 1991

26. Moss M, McLean D. Paediatric and adult computed tomography practice and patient dose in Australia. Australas Radiol 2006;50:33-40 